\title{
Motivación y satisfacción laboral del personal de Enfermería en el Hospital de Apoyo San Francisco, Ayacucho-2018
}

\author{
Motivation and job satisfaction of Nursing staff at the San Francisco Support \\ Hospital, Ayacucho-2018
}

\author{
Marleny Montes Salcedo', María Magdalena Díaz Orihuela²
}

\begin{abstract}
RESUMEN
Objetivo del estudio fue determinar la relación que existe entre el grado de motivación y el nivel de satisfacción laboral del personal de enfermería que labora en el Hospital de Apoyo San Francisco Ayacucho, 2018. Metodología: Tuvo un enfoque cuantitativo y de tipo correlacional, de diseño no experimental. El Instrumento para valorar la variable motivación fue trabajado por Hackman y Oldham (1974), y para evaluar la satisfacción laboral se usó la escala SL-SPC comprobado por la psicóloga Sonia Palma, ambos instrumentos se aplicaron a 50 personas que representan al personal de Enfermería. Resultados: Permitieron demostraron que entre la variable motivación y la variable satisfacción laboral muestran una relación moderada directa y significativa $(r=0.488, p=0.000)$, el análisis realizado entre la variable satisfacción laboral y las dimensiones de la motivación evidenció relación moderada y significativa en la retroalimentación $(r=0.425, p=0.002)$, y la autonomía para la realización de la tarea $(r=0.406, p=0.003)$; la importancia de la tarea reveló relación baja directa y significativa $(r=0.334, p=0.000)$.No se encontró suficiente evidencia para afirmar una relación entre la satisfacción laboral y las dimensiones identidad con la tarea $(r=0.251, p=0.079)$, y variedad de la tarea $(r=0.049$, $\mathrm{p}=0.736$ ). Conclusión: Que existe relación moderada entre la variable motivación y satisfacción laboral. Asimismo, se observa una relación moderada entre la dimensión retroalimentación y autonomía para la realización de la tarea.
\end{abstract}

Palabras clave: Motivación laboral, satisfacción laboral, enfermería.

\section{ABSTRACT}

Objective: Of determining the relationship that exists between the degree of motivation and the level of job satisfaction of the nursing staff working in the Hospital of Support San Francisco Ayacucho, 2018. Methodology: Has a focus quantitative and correlational type, non-experimental design. Instrument: to assess the motivation variable was worked by Hackman and Oldham (1974), and to evaluate job satisfaction the SL-SPC scale was used, verified by the psychologist Sonia Palma, both instruments were applied to 50 people representing the nursing staff. Results allowed to verify that the correlation between the variable motivation and the variable of job satisfaction show a moderate and direct relationship $(r=0.488, p=0.000)$, the analysis made between the variable of job satisfaction and the dimensions of the motivation showed a moderate relationship and significant in the feedback $(r=$ $0.425, p=0.002)$, and autonomy for the completion of the task $(r=0.406, p=0.003)$; the importance of the task revealed a direct and significant low relation $(r=0.334, p=0.000)$. Not enough evidence was found to affirm a relationship between job satisfaction and identity dimensions with the task $(r=$ $0.251, p=0.079)$ and variety of the task $(r=0.049, p=0.736)$. Conclusion: That there is a moderate relationship between the variable motivation and job satisfaction. Likewise, a moderate relationship is observed between the feedback dimension and the autonomy to carry out the task.

Keywords: Work motivation, job satisfaction, nurses

${ }^{1}$ Universidad Peruana Unión, Juliaca, Perú - Orcid ID: 0000-0003-4189-3418

${ }^{2}$ Clínica Adventista Ana Stahl, lquitos, Perú. 


\section{INTRODUCCIÓN}

La motivación se puede definir como la fuerza que hay en la persona, para satisfacer una necesidad, lo que empuja a realizar esa acción. Es importante para la gestión del sector salud la motivación en los trabajadores del hospital; es por eso que la rotación del personal de enfermería sirve como herramienta para saber la idoneidad del trabajador en relación a sus preferencias (Reynaga, 2015). Por otro lado, la satisfacción es el sentimiento de placer o bienestar, ésta se experimenta cuando se ha colmado un deseo o cuando se cubre una necesidad (Pablos, 2016). Asimismo, se considera la satisfacción como uno de los indicadores necesarios para promover la calidad en la atención, ya que el personal exteriorizará su sentir de acuerdo a las condiciones de trabajo en las que se desenvuelve (Cisneros, 2011).

Las organizaciones de salud muestran estrategias continuas y sistematizadas para estimar las condiciones en las que se brinda la atención al usuario y/o cliente, la cual está cercanamente involucrada con la satisfacción del prestador del servicio. El personal de salud no desarrollará su trabajo con calidad, con eficiencia si no se encuentra satisfecho durante su desempeño laboral. De igual modo, es necesario tomar en cuentalas expectativas personales y profesionales para establecer un diagnóstico situacional que permita identificar los problemas y el diseño de acciones a mejorar, con la finalidad de enmendar aquellos aspectos que sean necesarios (Pavón , Blázquez Sobeida, y Blázquez, 2011).El nivel de satisfacción que experimenta el colaborador, en términos de bienestar deseable se verá reflejado en la calidad y la productividad en cualquier tipo de profesión (Chiang, Salazar y Núñez, 2007).

Los trabajos relacionados con el sector salud se caracterizan por algunos estresores que resultan de una actividad impetuosa en la dedicación al cuidado de la vida humana; en este sentido, el compromiso por la vida, las relaciones empático y afectivas con el paciente y las características propias de las instituciones sanitarias, colocan a los trabajadores de la salud en una situación de riesgo permanente. Esta tensión cotidiana aumenta aún más si los profesionales desempeñan sus funciones en instituciones sanitarias de países en vías de desarrollo. En el ámbito de las instituciones públicas, el recorte de las partidas presupuestarias, la insuficiencia de la infraestructura hospitalaria, la falta de medicamentos y la disminución de los salarios profesionales, configuran un clima laboral inestable y estresante (Camponovo Meier \& Morín Imbert, 2000; citado por Paris y Omar, 2008).El grado de satisfacción laboral influenciará en el tiempo dedicado a la vida laboral, la disposición para realizar un conjunto de actividades, la tendencia a abandonar el centro de labor.La relevancia de la motivación y satisfacción en el trabajo como temas estudiados desde hace mucho, se debe a los efectos que se perciben en la vida del trabajador y su implicancia en la empresa en relación a la disposición del mismo para realizar los trabajos con un alto grado de cumplimiento. (Moya, 2011).

\section{METODOLOGÍA}

El presente estudio tiene un enfoque cuantitativo, de tipo correlacional por que se miden dos variables y establecen una relación estadística entre las mismas y de diseño no experimental, porque no existe intervención de ningún tipo por parte del investigador (Hernández, 2014).

\section{Participantes.}

La población de estudio estuvo compuesta por 70 personas que constituyen el personal de enfermería que laboran en los diferentes servicios tanto consultorios externos como hospitalización de Hospital de Apoyo San Francisco. El tipo de muestreo fue no probabilístico por conveniencia por las características de muestra, haciendo un total de 50 personas que representan al personal de enfermería (26 enfermeras y 24 técnicos). Teniendo en cuenta los criterios de inclusión y exclusión.

\section{Instrumentos}

El instrumento de medición de la variable motivación fue diseñado por Hackman y Oldham (1974), quien estudió cinco dimensiones: variedad de habilidades, identidad de la tarea, significación de la tarea, autonomía y retroalimentación del puesto, que consta de 23 preguntas de respuesta tipo Likert: totalmente de acuerdo 5 , de acuerdo 4 , indeciso 3 , en desacuerdo 2 y totalmente en desacuerdo 1. La fiabilidad fue determinada mediante el Coeficiente Alpha de Cronbach en una muestra piloto de 10 profesionales de Enfermería del Hospital de Apoyo de Jesús Nazareno. Una confiabilidad de 0,80 asegura 
la adecuada precisión del instrumento (Montes, 2015). Y la escala SL-SPC que fue validado por la psicóloga Sonia Palma Carrillo para Satisfacción laboral, que consta de 36 preguntas, con respuestas tipo Likert. La confiabilidad fue de 0.917 según el coeficiente Alfa de Cronbach

\section{Análisis de datos}

Se utilizó la prueba estadística de correlación de Pearson. IBM, SPSS Statics versión 25.

\section{RESULTADOS Y DISCUSIÓN}

La tabla 1 muestra que el $54 \%$ del personal de enfermería tienen motivación media, seguido por el $36 \%$ del personal que tiene motivación alta y sólo el 10\% tiene motivación baja. En la investigación realizada por Antoño y Santiago (2015) Motivación y satisfacción laboral del profesional enfermero (a) del Hospital II Ramón Castilla Es Salud. Los resultados globales muestran que el $57 \%$ (20) de las enfermeras(os) presentan motivación media. En tanto otro autor Montes (2015) en su estudio encontró que del $100 \%$ de profesionales de Enfermería del Hospital Regional de Ayacucho, el $30,4 \%$ presenta bajo nivel de motivación y predominó en $24,7 \%$ la insatisfacción laboral. El $17,1 \%$ presenta alto nivel de motivación y todos ellos reportan la satisfacción laboral. Concluye el autor evidenciando: cuanto mayor es el nivel de motivación es mayor la satisfacción laboral, porque la motivación incide en el comportamiento de los empleados. Sin embargo (Rueda, 2014) en su estudio "Motivación del personal de enfermería para la calidad de cuidado en usuarios y usuarias" llegó a las siguientes conclusiones: la tendencia de las respuestas de la población estudiada está ubicada en la alternativa "poco motivado(a)" con una tasa de 64 por ciento $(n=13)$; continuando de un 23.6 por ciento $(n=5)$ que correspondió a la opción "moderada motivación". La tasa más baja (1.8\%), se presentó en la alternativa motivado(a); observándose ninguna respuesta en la opción "altamente motivado(a)". Se debe destacar que el cuidado humano de calidad es oportuno porque es producto entre otras de la motivación que el personal posea dentro de su área de trabajo, en este sentido Locke (2000) señala que en el ambiente laboral debe existir un equilibrio entre los factores motivacionales intrínsecos y extrínsecos, con el finalidad de que el personal que labore dentro de una institución ya sea de salud o no, presente una respuesta positiva y satisfactoria y según las características físicas y de personalidad que posean, deben ser asignados a un área donde desempeñen de manera eficaz las actividades o las competencias que se requiera, lo cual dará una respuesta positiva tanto para la institución como para el personal que allí labore.

Tabla 1

Motivación del personal de enfermería del Hospital de Apoyo San Francisco - Ayacucho, 2018

\begin{tabular}{lll} 
Motivación & $\mathrm{n}$ & $\%$ \\
Baja & 5 & 10.0 \\
Media & 27 & 54.0 \\
Alta & 18 & 36.0 \\
Total & 50 & 100 \\
\hline
\end{tabular}

La tabla 2 muestra que el $84 \%$ de los profesionales que participaron en el estudio tiene mediana satisfacción laboral, $10 \%$ alta satisfacción laboral, y $6 \%$ baja satisfacción laboral; no se encontró ningún participante en las categorías sin satisfacción laboral y satisfacción laboral muy alta. Si hacemos una comparación entre el nivel de motivación y satisfacción ambos tienen los resultados de mediana motivación y mediana satisfacción, es decir existe la mayor probabilidad de que cuanto mayor es la motivación laboral será mayor la satisfacción del personal de Enfermería del Hospital de Apoyo San Francisco. En cuanto a la satisfacción también se cuenta con estudios que coinciden Moya (2011) en su estudio obtuvo que el $67,4 \%$ de respuestas con una media de satisfacción. Las fuentes que generan más satisfacción han resultado ser las relaciones con los compañeros, el propio trabajo y la competencia profesional. Por el contrario, los encuestados se sentían insatisfechos con el reconocimiento, la promoción profesional y la tensión relacionada con el trabajo. Se encuentran diferencias significativas entre el personal fijo de mañana y a turnos, el personal fijo de plantilla y los contratos temporales, y en relación al tiempo trabajado. Asimismo Contreras (2013) en su estudio "Satisfacción laboral de los profesionales de enfermería vinculados a una I.P.S de III nivel de atención Bogotá 2013", llega a los siguientes resultados que el $58 \%$ de la población presenta un nivel medio de satisfacción laboral. Seguido 
del $31 \%$ de la población que presenta un nivel alto de satisfacción laboral y por último el $11 \%$ de la población con un nivel bajo de satisfacción laboral. Por otro lado Aguilar (2010)para ello se identificaron caracterlu00edsticas sociodemogrl u00e1ficas, y caracterlu00edsticas laborales del personal de enfermerlu00eda. Materiales y MI u00e9todos: Se trato de un estudio descriptivo multiclu00e9ntrico cuya muestra la conformaron 218 enfermeras que laboran en el sector pl u00fablico y privado de las Cllu00ednicas y Hospitales de Bucaramanga y su lu00e1rea Metropolitana. Se apliclu00f3 el cuestionario de satisfaccilu00f3n laboral S10/12 de J.L Melil u00e1 y J.M.Peiro (1998 menciona que el grado de satisfacción puede afectar la cantidad y calidad del trabajo que desempeñan los sujetos, además de incidir sobre otros aspectos como la baja calidad de la prestación, el ausentismo laboral, la propensión a abandonar la organización, etc. El personal de enfermería del Hospital de Apoyo San Francisco, tiene en consideración que trabaja en una institución cuya finalidad es prestar servicios de salud que contribuya con la satisfacción de la necesidad de salud de las personas; muchos de estos profesionales trabajan en ambientes muy desfavorables, brindando cuidados críticos enfrentando conflictos emocionales y no pocos laborales diariamente; sin embargo están satisfechos, al respecto (Herzberg, 1977) manifiestas de que hay factores que están involucrados con la satisfacción interna, es decir tienen que ver con el contenido del cargo y la naturaleza de las tareas que el personal ejecuta, están bajo el control del individuo pues se relaciona con aquello que él hace y desempeña, que tienen que ver con crecimiento, desarrollo personal, autorrealización y estos producen un efecto de satisfacción duradera y sostenible.

Tabla 2

Satisfacción laboral del personal de enfermería del hospital de apoyo San Francisco - Ayacucho, 2018

\begin{tabular}{lll} 
Satisfacción & $\mathrm{n}$ & $\%$ \\
\hline Sin satisfacción laboral & 0 & 0.0 \\
& & 6.0 \\
Baja satisfacción laboral & 3 & 84.0 \\
Mediana satisfacción laboral & 42 & 10.0 \\
Alta Satisfacción laboral & 5 & 0.0 \\
Satisfacción laboral muy alta & 0 & 100 \\
Total & 50 & \\
\hline
\end{tabular}

En la tabla 3: El análisis de correlación entre la motivación y la satisfacción laboral muestra una relación moderada directa y significativa $(r=0.488$, $p=0.000$ ), el análisis realizado entre la satisfacción laboral y las dimensiones de la motivación evidenció relación moderada y significativa en la retroalimentación $(r=0.425, p=0.002)$, y la autonomía para la realización de la tarea $(r=0.406$, $\mathrm{p}=0.003)$; la importancia de la tarea mostro relación baja directa y significativa $(r=0.334$, $p=0.000$ ). No se encontró evidencia suficiente para afirmar una relación entre la satisfacción laboral y las dimensiones identidad con la tarea $(r=0.251$, $p=0.079)$, y variedad de la tarea $(r=0.049, p=0.736)$. coincide con algunos estudios: Antoño \& Santiago, (2015) concluyeron que existe relación entre el nivel de motivación y la satisfacción Laboral del profesional de Enfermería del Hospital II Ramón Castilla. Tau C Kendal: Valor: 0,573. Asimismo Vásquez (2007) en su investigación logró mostrar que existe relación entre el nivel de motivación y la satisfacción Laboral del profesional de Enfermería. De la misma forma Zubiri (2013) refiere que la motivación y la satisfacción, son elementos fundamentales para el éxito organizacional, siendo que de ella depende la consecución de los objetivos. Asimismo, el autor se refiere que muchos sectores, entre ellos el sanitario, no se han percatado aun de la importancia de estas cuestiones y sus dirigentes siguen practicando una gestión que no tiene en cuenta el factor humano. Estas circunstancias podrían acarrear un déficit de recursos calificados a largo plazo, con la consiguiente disminución de la calidad asistencial. En este contexto, resulta imprescindible que las gerencias analicen de forma periódica aquellos factores distorsionantes, tanto de la motivación como de la satisfacción laboral, entre los que se encuentran la supervisión, las políticas de empresa y las condiciones de trabajo. 
Marin y Placencia (2016) en su investigación evidenció que existe correlación nula y no significativa entre motivación laboral y la satisfacción laboral del personal .Por tanto, se determina que existe correlación positiva (0.336) entre ambas variables aunque de baja intensidad, lo cual nos permite aseverar que $\mathrm{A}$ mayor grado de motivación laboral, mayor grado de satisfacción laboral del personal de Socios en Salud Sucursal Perú.

Finalmente en relación a las dimensiones de la Motivación que son Variedad de la tarea, identidad de la tarea, importancia de la tarea, autonomía de la realización de la tarea y la retroalimentación y su relación con la satisfacción laboral se logra mostrar que: el análisis realizado entre la satisfacción laboral y las dimensiones de la motivación evidenció relación moderada y significativa en la retroalimentación $(r=0.425, p=0.002)$, y la autonomía para la realización de la tarea $(r=0.406, p=0.003)$; la importancia de la tarea mostro relación baja directa y significativa $(r=0.334, p=0.000)$. No se encontró evidencia suficiente para afirmar una relación entre la satisfacción laboral y las dimensiones identidad con la tarea $(r=0.251$, $p=0.079)$, y variedad de la tarea $(r=0.049$, $p=0.736$ ). Podemos concluir que las dimensiones que generan una motivación media en el personal de enfermería se caracterizaron por la retroalimentación donde reciben información clara y directa sobre sus funciones y tareas que les corresponde, asimismo por la autonomía ya que la responsabilidad de su trabajo les proporciona libertad, toma de decisiones con grados de libertad generados por sus habilidades y competencias. Hay estudios que coinciden con nuestros datos como el de Antoño \& Santiago (2015) Motivación y satisfacción laboral del profesional enfermero (a) del Hospital II Ramón Castilla Es Salud. Al analizar esta variable según dimensiones, se encontró que en las dimensiones: importancia, retroalimentación y autonomía presentan niveles medio con tendencia a alta, mientras que las dimensiones identidad y variedad de la tarea presentan mayoritariamente nivel bajo. En la satisfacción laboral, el 63\% (22) de enfermeros tienen nivel medio. $Y$ según dimensiones se encontró que más del $50 \%$ de dichos profesionales presentan nivel medio, en Relaciones sociales, Desempeño de tareas y Relación con la autoridad, Políticas administrativas y Desarrollo personal presentan nivel medio con tendencia a nivel alto y en los factores Beneficios Laborales y Condiciones físicas y/o confort niveles de satisfacción que entre medio y bajo. Se concluye que existe relación entre el nivel de motivación y la satisfacción Laboral del profesional de Enfermería del Hospital II Ramón Castilla Tau C Kendal: Valor: 0,573. Sin embargo Aguilar(2010)para ello se identificaron caracterlu00edsticas sociodemogrl u00e1ficas, y caracterlu00edsticas laborales del personal de enfermerlu00eda. Materiales y MI u00e9todos: Se trato de un estudio descriptivo multiclu00e9ntrico cuya muestra la conformaron 218 enfermeras que laboran en el sector pl u00fablico y privado de las Cllu00ednicas y Hospitales de Bucaramanga y su lu00e1rea Metropolitana. Se apliclu00f3 el cuestionario de satisfaccilu00f3n laboral S10/12 de J.L Melii u00e1 y J.M.Peiro (1998menciona que estudios han demostrado que una manera de motivación en el trabajo se da con el enriquecimiento de tareas, también llamado enriquecimiento de cargos, el cual consiste en la sustitución de las tareas más simples y elementales del cargo por tarea más compleja, que ofrezcan condiciones de desafío, de retos y de satisfacción personal.

Vasquez (2007) en su investigación obtuvo los siguientes resultados: que el $25(48 \%)$ de las enfermeras(os) presentan motivación media. Asimismo al valorar las dimensiones de la motivación se obtuvo que más de $50 \%$ de los profesionales presentan motivación media; destacándose las dimensiones identidad y autonomía por mostrar niveles significativos de motivación media con tendencia a alta, en cuanto a las dimensiones que presentan nivel bajo son retroalimentación, importancia y variedad de la tarea. Con respecto a la satisfacción en el entorno laboral el $28(53.8 \%)$ de las enfermeras(os) tienen nivel medio, en relación a los factores determinantes de la satisfacción laboral se mostró que más del $40 \%$ de dichos profesionales presentan nivel medio, destacando los factores Desempeño de tareas, Relación con la autoridad y Beneficios laborales y remunerativos por mostrar niveles Significativos de satisfacción media, las dimensiones Relaciones interpersonales, Desarrollo personal y Políticas administrativas presentan tendencia al nivel alto y el factor Condiciones físicas y/o confort presenta niveles de satisfacción que oscilan entre alta y baja. Al aplicar Ji - Cuadrado se obtuvo X2 Calculado > X2 Tabulado ello permitió rechazar la Ho y aceptar la hipótesis del estudio que afirma existe relación entre el nivel de motivación y la satisfacción Laboral del profesional de Enfermería. 
Tabla 3

Relación entre la motivación y sus dimensiones con la satisfacción laboral de los profesionales de enfermería del hospital de apoyo San Francisco - Ayacucho, 2018

\begin{tabular}{llll} 
Variables y dimensiones & Satisfacción & & \\
Motivación & $n$ & $r$ & 0.000 \\
$\quad$ Dimensiones & 50 & 0.488 & 0.736 \\
\hline Variedad de la tarea & & & 0.079 \\
Identidad con la tarea & 50 & 0.049 & 0.018 \\
Importancia de la tarea & 50 & 0.251 & 0.003 \\
\hline Autonomía para la realización de la tarea & 50 & 0.334 & 0.406 \\
\hline
\end{tabular}

\section{CONCLUSIÓN}

En cuanto a la correlación de la motivación y la satisfacción laboral muestra una relación moderada directa y significativa $(r=0.488$, $p=0.000$ ).

En cuanto al nivel de motivación la mayoría de los encuestados tienen una motivación de nivel medio, seguido de motivación alta. $Y$ con respecto al nivel de satisfacción la mayoría de los profesionales que participaron en el estudio tiene mediana satisfacción laboral, seguido de alta satisfacción laboral en poca cantidad; no se encontró ningún participante en las categorías sin satisfacción laboral y satisfacción laboral muy alta. Al analizar esta variable según dimensiones, se encontró que en las dimensiones: importancia, retroalimentación y autonomía presentan niveles medio con tendencia a alta, mientras que las dimensiones identidad y variedad de la tarea presentan mayoritariamente nivel bajo.

Declaración de financiamiento y de conflictos de interés:

El estudio fue financiado por los autores, quienes declaran no tener conflictos de interés

\section{Correspondencia}

Marleny Montes Salcedo

Correo electrónico:

marlenymontes@upeu.edu.pe

\section{María Díaz Orihuela}

Correo electrónico:

magi@upeu.edu.pe

\section{REFERENCIAS BIBLIOGRÁFICAS}

Aguilar S. (2010). Satisfacción laboral en profesionales de enfermería. Cuidarte, 1(1), 5362. Recuperado de https://doi.org/http://dx.doi. org/10.15649/cuidarte.v1i1.74

Antoño N, \& Santiago C. (2015). Motivacion y satisfacción laboral del profesional enfermero (a) del Hospital IIRamon Castilla Es Salud. Universidad Privada Norbert Wiener. Recuperado de http://repositorio.uwiener.edu.pe/ handle/123456789/309

Calcina M. (2012). Clima organizacional y satisfacción laboral en enfermeros de unidades críticas del Hospital Nacional Dos de Mayo 2012. Universidad Nacional Mayor de San Marcos. Recuperado de. http://ateneo.unmsm.edu.pe/ ateneo/bitstream/123456789/4937/1/Calcina_ Caceres_Marcos_Herminio_2015.pdf

Chiang M., Salazar, C. M., \& Núñez A. (2007). Clima organizacional y satisfacción laboral en un establecimiento de salud estatal: hospital tipo 1. Theoria, 16(2), 61-76. Recuperado de http:// www.redalyc. org/articulo.oa? $\mathrm{id}=29916206$

Chiavenato I. (2009). Gestión del talento humano. (McGraw-Hill, Ed.) (1ra.). Mexico.

Cisneros C. (2011). Satisfacción Laboral del Personal de Enfermería y su Relación con las Condiciones de Trabajo Hospitalario. Universidad Autónoma de San Luis de Potosí Recuperado de http://repositorio.ucv.edu.pe/bitstream/handle/ UCV/1083/reyes_oa.pdf?sequence=1

Contreras M. (2013). Satisfacción laboral de los profesionales de enfermería vinculados a una I.P.S de III nivel de atención Bogotá 2013. Universidad Nacional de Colombia. Universidad Nacional de Colombia. Recuperado de http://www.bdigital. unal.edu.co/43104/1/33104241.2013.pdf 
Davis K, \& Newstrom J. (2003). Comportamiento humano en el trabajo. (McGraw-Hill, Ed.) (1ra. ed). Mexico.

Daza D. \& P. C. (2007). Importancia de la satisfaccion laboral en los empleados de las organizaciones. Universidad Tecnologica de Bolivar. Recupeado de http://biblioteca.unitecnologica.edu.co/notas/ tesis/0042607.pdf

Hackman R, y Oldham G. (1974). The Job Diagnostic Survey: An Instrument for the Diagnosis of Jobs and the Evaluation of Job Redesign Projects. Doctoral Thesis. Recuperado de. https://doi. org/10.1111/j.1466-7657.2007.00507.x

Hernandez R. (2014). Metodologia de la Investigación. S. A. d. C. V. Interamericana editores, Ed, Mc Graw Hill (6ta edició, Vol. 91). Mexico.

Herzberg F. (1977). Una vez más: ¿Cómo motivar a los trabajadores? Volumen 5 de Harvard Business Review, 1977.

Montes M. (2015). Nivel de motivación y satisfacción laboral de profesionales de Enfermería del Hospital Regional. Universidad Cesar Vallejo. Recuperado de: http://repositorio.ucv.edu. pe/bitstream/handle/UCV/20454/navarro_ vl. pdf? sequence=1\&isAllowed=y

MarinH, \&PlacenciaM.(2016). Motivaciónysatisfacción laboral del personal de una organización de salud del sector privado. HORIZONTE MEDICO, 17(4), 42-52. Recuperado de http:// www.scielo.org.pe/scielo.php? pid=S1727558X2017000400008\&script=sci_abstract

Morillo I. (2006). Nivel de satisfacción del personal académico del Instituto Pedagógico de Miranda José Manuel Sisso Martínez en relación con el estilo de liderazgo del jefe del departamento. Sapiens, 7(1), 43-57. Recuperado de https:// www.redalyc.org/pdf/410/41070104.pdf

Moya S. (2011). Motivación y Satisfacción de los profesionales de Enfermería. Investigación aplicada en un área quirurgica. Universidad de Cantabria. Recuperado de: https://repositorio. unican.es/xmlui/handle/10902/477.

Pablos M. (2016). Estudio de Satisfacción Laboral y Estrategias de Cambio de las Enfermeras en los Hospitales Públicos de Badajoz y Cáceres. Universidad de Extremadura. Recuperado de: http://www.areasaludbadajoz.com/images/datos/ docencia_e_investigacion/419drh.pdf

Palma S. (2005). Escala de Satisfacción Laboral (SLSPC). (C. EIRL, Ed.). Lima Perú.

Paris L., \& Omar, A. (2008). Predictores de satisfacción laboral en médicos y enfermeros. Estudos de Psicologia, 13(3), 233-244.
Recuperado de. https://doi.org/10.1590/S1413294X2008000300006

Pavón P, Carmen, G. M. del, Blázquez S, \& Blázquez C. (2011). Satisfaccion del Personal de Salud en un Hospital Universitario. Salud en Tabasco, 17, 13-21. Recuperado de http://www.redalyc.org/ pdf/487/48721182003.pdf.

Perez L. (1993). Fundamentos de la direccion de empresas. (Rialp). Madrid España.

Perez P. (2011). Una revisión actual de la aplicación del concepto de Satisfaccion laboral y su evaluaciónhacia un modelo integrador. Recupeado de https:// www.redalyc.org/jatsRepo/1941/194154512012/ html/index.html

Pinto L, \& Portugal E. (2016). Factores del Ejercicio Profesional Relacionados a la Satisfacción laboral de las Enfermeras Centro Quirurgico del Hospital Regional Honorio Delgado. Universidad Nacional de San Agustín de Arequipa. Recuperado de http://repositorio.unsa.edu.pe/bitstream/handle/ UNSA/1804/ENpisal.pdf?sequence $=1$

Reynaga Y. (2015). Motivación y desempeño laboral del personal en el Hospital Hugo Pesce Pescetto de Andahuaylas. Universidad Nacional José Maria Arguedas. Recuperado de http://repositorio.unajma.edu.pe/bitstream/ handle/123456789/245/Yolanda_Reynaga_ Tesis_Titulo_2016.pdf?sequence $=1$ \&isAllowed $=\bar{y}$

Robinns S. (1999). Fundamentos del comportamiento organizacional. (Prentice Hall, Ed.) (8va. ed). Mexico.

Rueda Y. (2014). Y usuarias Resumen Introducción El problema. Universidad Nacional Experimental de los LLanos Centrales Romulo Gallegos. Maracay-Venezuela.. Recupeado de https:// www.researchgate.net/institution/Universidad_ Nacional_Experimental_de_los_Llanos Centrales_Romulo_Gallegos

Santander P. (2012). Desempeño laboral de los enfermeros (a) egresados de la Universidad Francisco de Paula Santander. Ciencia y Cuidado, 2(4). Recupeado de https://revistas.ufps.edu.co/ index.php/cienciaycuidado/article/view/340

Vasquez S. (2007). Nivel de motivación y su relación con las satisfacción laboral del profesional de enfermería en el Hospital Nacional Arzobispo Loayza, 2006. Universidad Nacional Mayor de San Marcos. Recuperado de http://cybertesis. unmsm.edu.pe/handle/cybertesis/488

Ventura S. (2012). Satisfacción laboral en docentesdirectivos con sección a cargo de cuatro instituciones de educación inicial pertenecientes a la UGEL 07. Pontificia Universidad Catolica del 
Perú..Recuperado de http://tesis.pucp.edu.pe/ repositorio/handle/20.500.12404/4734

Vera V, Inga C, y Atamari N. (2013). Satisfacción laboral del personal de salud en una zona de conflicto armado de Cusco, Perú. Anales de La Fultad de Medicina, 76, 1-3

Zelada V. (2014). Nivel de satisfacción laboral del personal asistencial del Centro de Salud Conde de la Vega Baja 2014. Universidad Nacional Mayor de San Marcos. Recuperado de http://pesquisa. bvsalud.org/portal/resource/pt/lil-782308.

Zubiri F. (2013). Satisfacción y motivación profesional. EDITORIAL, 36, 193-196.

Recibido: 3 de marzo

Aceptado: 7 de julio 